\title{
Scolarité « protégée » et abandon scolaire
}

Les paradoxes norvégiens

"Protected" schooling and school dropout. The Norwegian paradoxes.

Escolaridad "protegida" y abandono escolar. Las paradojas noruegas

\section{Anne-Lise Høstmark Tarrou}

\section{OpenEdition} Journals

Édition électronique

URL : https://journals.openedition.org/ries/2098

DOI : 10.4000/ries.2098

ISSN : 2261-4265

\section{Éditeur}

France Education international

\section{Édition imprimée}

Date de publication : 1 septembre 2011

Pagination : 109-118

ISBN : 978-2-85420-592-3

ISSN : $1254-4590$

Référence électronique

Anne-Lise Høstmark Tarrou, « Scolarité « protégée » et abandon scolaire », Revue internationale d'éducation de Sèvres [En ligne], 57 | septembre 2011, mis en ligne le 01 septembre 2014, consulté le 06 juillet 2021. URL : http://journals.openedition.org/ries/2098 ; DOI : https://doi.org/10.4000/ries. 2098 


\section{Scolarité "protégée " et abandon scolaire}

\section{Les paradoxes norvégiens}

\section{Anne-Lise Høstmark Tarrou}

Le plaisir à l'école, dans le contexte socioculturel norvégien, semble lié au plaisir, pour chaque élève, de se sentir à l'aise, accepté par les autres élèves et mis en valeur par les enseignants. Il semble également important que ce que l'école demande aux élèves d'apprendre ait un sens pour eux, et que cela leur inspire la joie de venir à l'école. Pour les élèves, les parents et les enseignants comme pour la société de façon plus large, l'idée qui prédomine est que les élèves doivent se sentir au cœur des apprentissages, et que le rôle de l'enseignant est avant tout de faciliter ces apprentissages en vue de favoriser l'épanouissement des élèves. Par l'analyse des textes qui définissent les objectifs généraux de l'éducation, des débats publics et en m'appuyant sur quelques recherches récentes, je vais tenter de cerner la façon dont le plaisir et l'ennui se manifestent chez les élèves norvégiens comme ailleurs, aux différents niveaux ${ }^{1}$ de la scolarité.

\section{DU CONSENSUS AU DÉBAT SUR LES VALEURS FONDAMENTALES Le discours institutionnel depuis 1974}

Un examen rapide des efforts institutionnels menés en Norvège pour que tous les élèves se sentent à l'aise à l'école montre que l'objectif principal, au cours des quarante dernières années, a été d'effacer les inégalités entre élèves à l'école comme dans la société (Tarrou 2001).

Les valeurs susceptibles de créer un climat scolaire qui facilite le plaisir et évite l'ennui à l'école, pour les élèves comme pour les enseignants, sont formulées dans les documents officiels depuis 1974, mais sont articulées avec plus de fermeté dans la réforme de 1994, avec l'établissement d'un plan cadre général pour toute la scolarité. Ce plan a été maintenu dans la nouvelle réforme mise en place à partir de 2006, intitulée "Promouvoir les connaissances ».

Prendre les mesures intelligentes pour que les élèves réussissent à l'école est une préoccupation primordiale et ancienne dans les discours institutionnels norvégiens. Pour faciliter le parcours des élèves tout au long des trois niveaux

\footnotetext{
1. L'enseignement primaire et le collège forment dix années de scolarité de base, obligatoire. Le lycée comprend trois années. Les filières techniques et professionnelles sont organisées sur la base de deux années au sein de la structure scolaire et d'une année d'apprentissage en entreprise, le plus souvent étalée sur deux ans, qui permet la participation à l'activité de production de l'entreprise.
} 
de l'enseignement, tout obstacle formel qui pourrait empêcher les élèves de suivre leur classe d'âge d'un niveau à l'autre a été supprimé.

C’est pourquoi, depuis les années soixante-dix, la notation des élèves est introduite tardivement dans la scolarité. L'accent est surtout mis sur l'égalité d'accès à l'école, indépendamment des origines sociales ou géographiques. Le tronc commun de scolarité, obligatoire jusqu'au collège inclus, a été mis en place en 1969. Depuis les années soixante-dix, l'égalité entre élèves issus de différentes classes sociales, entre les sexes, entre élèves à besoins pédagogiques spécifiques, et l'égalité entre les différentes voies d'enseignement et de formation sont également devenues des valeurs constitutives au lycée. En 1994, le parlement a légiféré pour donner à tous les jeunes âgés de 16 à 20 ans le droit de suivre un enseignement au lycée (Tarrou, 2001, p. 105-122).

L'institutionnalisation de la formation technique et professionnelle, en 1974, fut une combinaison entre formation dans les écoles et en entreprise, en collaboration entre les partenaires sociaux et les autorités de tutelle. Avec la réforme de tout l'enseignement et de la formation en 1994, la formation technique et professionnelle initiale fut intégrée à l'enseignement secondaire, sur la base d'un plan cadre commun.

Les objectifs du plan cadre général de base pour tout l'enseignement norvégien, voté pour la première fois en 1994, ont été formulés sous forme d'objectifs qualitatifs pour former les « sept hommes » suivants :

- l'homme en recherche de sens, tourné vers les valeurs chrétiennes et humanistes, son héritage culturel et son identité ;

- l'homme créatif, qui développe ses dons, et met l'accent sur le travail créatif, la recherche, et le "vécu »;

- l'homme travailleur, tourné vers les apprentissages et le travail, qui valorise le rôle des formateurs;

- l'homme de préoccupé de culture générale, qui recherche les cadres globaux de références et de compréhension commune dans une société spécialisée ;

- l'homme de médiation, qui s'appuie sur la culture des élèves, la participation des parents d'élèves et la société locale ;

- l'homme conscient de la nécessité de respecter l'environnement ;

- l'homme intégré, c'est-à-dire celui qui réunit toutes ces qualités ${ }^{2}$.

Ces objectifs expriment les finalités de l'ensemble de la scolarité norvégienne, de façon que les élèves puissent se développer dans un climat de sécurité, qui donne du sens à ce qu'ils apprennent et qui contribue à leur épanouissement (Tarrou 2003, p. 133).

2. À titre d'exemple, on peut citer : « La formation doit contribuer à créer/à former des caractères, pour que chacun puisse prendre en charge sa propre vie, ses obligations vis-à-vis la société et adopter une attitude de respect de l'environnement »; " L'homme doit être à même de rompre avec l'égocentrisme et l'adhésion à la loi du plus fort, l'enseignement et la formation doivent donner aux jeunes la force de rester autonomes, de trouver seuls leur route et de ne pas accepter sans examen critique l'opinion des autres ». 
Ce plan cadre a été maintenu pour l'ensemble de la scolarité obligatoire et pour le lycée, dans la réforme de 2006 toujours en vigueur. L'idée est qu'il imprègne les plans d'études et la mise en place des contenus et des modes de travail à tous les niveaux scolaires, tout en prenant en compte les besoins des enfants et les intérêts des enseignants ${ }^{3}$. Cette dernière réforme, initiée sur la base d'un rapport du ministère de l'Éducation intitulé "La culture pour l'apprentissage » (St.meld. 30, 2003-2004), souligne le lien étroit entre la culture du pays et les apprentissages. Le rapport constitua la base d'une réforme profonde de la structure et du contenu de l'ensemble de l'enseignement en 2006, intitulée " Promouvoir les connaissances». Cette réforme souligne l'importance de renforcer le développement des connaissances à tous les niveaux de la scolarité. La réforme en est encore à ses débuts et des recherches commencent à être publiées sur sa mise en place (Tarrou 2009, p. 103-120, Tarrou et Johannesen 2010, p. 93-107).

Ces valeurs fondamentales de l'enseignement norvégien influencent fortement, selon moi, la façon dont les Norvégiens pensent le plaisir et l'ennui à l'école. Elles expriment la politique éducative norvégienne et influencent également le débat public actuel sur le "décrochage " ou le manque d'intérêt des élèves pour l'école (Tarrou, 2003, p. 132-133).

Malgré toutes les mesures mises en place, des inégalités et des mécontentements perdurent encore chez les élèves comme chez les enseignants. Nous allons étudier la façon dont cela se manifeste dans les pratiques.

\section{Une crise des certitudes depuis 2006 ?}

Le débat public norvégien actuel concerne avant tout les effets de la mise en place de la réforme de 2006. Les problèmes les plus fréquemment soulevés, et qui sont fortement liés aux questions du plaisir et de l'ennui chez les élèves, sont l'abandon scolaire et le désintérêt pour l'école chez un certain nombre de jeunes collégiens et lycéens. Un débat plus délicat sur la notation dans la scolarité obligatoire semble aussi émerger de nouveau dans certains milieux scolaires, notamment à Oslo. Depuis la publication des rapports PISA et TIMMS, ce débat a refait surface récemment en raison des résultats des élèves norvégiens en lecture et en mathématique. La Norvège se situant au milieu du classement dans la dernière étude PISA, les journaux, les partis politiques, et les chercheurs débattent de l'opportunité d'introduire la notation des élèves à partir de la huitième année de la scolarité, au lieu d'attendre la dixième année, en fin de collège. Certains politiques sont convaincus que l'absence de notation jusqu'à la dixième année de la scolarité obligatoire est la cause de la faiblesse des résultats norvégiens dans les évaluations internationales.

3. Dans la mesure où les plans d'études sont des plans cadres pour chaque filière, les enseignants disposent d'une large marge de manœuvre afin de prendre en compte les besoins des élèves. 
Ce débat entraîne un autre débat, encore plus intéressant, sur la relation entre l'absentéisme des élèves au collège et la réussite des élèves au lycée.

Pour comprendre le contexte dans lequel ce débat a lieu, il faut se souvenir qu'un débat particulièrement vif, au cours des années soixante-dix, a conduit à l'abolition, en 1976, de la notation jusqu'à la fin du collège. Depuis, la Norvège n'était pas revenue sur ce point. L'un des grands arguments soulevés à l'époque en faveur de l'absence de notation fut de ne pas stigmatiser les jeunes par un classement par des notes, qui risquait de traumatiser quelques uns et de favoriser d'autres.

\section{LE PLAISIR ET L'ENNUI DANS LES TROIS NIVEAUX D'ENSEIGNEMENT}

Nous allons maintenant présenter par niveau de scolarité ${ }^{4}$ la façon dont les bonnes idées inscrites dans les documents officiels sont mises en pratique, en ce qui concerne le plaisir et l'ennui.

\section{Le plaisir et l'ennui dans l'enseignement primaire}

L'enseignement primaire comprend le niveau élémentaire pour les élèves âgés de 6 à 10 ans, et le niveau moyen pour les élèves âgés de 11 à 13 ans. Cet enseignement est sans notation, sans redoublement et sans échec scolaire, donc sans obstacles structurels susceptibles de perturber le climat de l'apprentissage. Dans ce sens, les enfants norvégiens jouissent d'une scolarité assez protégée des obstacles formels susceptibles de provoquer de l'angoisse chez les enfants. L'idée sous-jacente est que les élèves, dans les premières années de leur scolarité, ne doivent pas être soumis à la perspective de l'échec à l'école, et que les enfants pourraient se sentir fragilisés et angoissés s'ils devaient s'inquiéter d'échouer au lieu de travailler dans un climat environnant de sécurité. Si les contraintes formelles n'existent donc pas pour les élèves de l'enseignement primaire en Norvège, un certain nombre d'élèves peuvent cependant connaître des difficultés extrascolaires, qui influent sur leur scolarité.

\section{Le collège}

Au niveau du collège, de la huitième à la dixième année, toujours à l'intérieur du tronc commun de la scolarité de base obligatoire, la situation devient différente pour les jeunes Norvégiens. Mais il n'existe toujours pas de notation des élèves avant la fin de ce niveau. Pendant ces trois années, les élèves 
sont toujours épargnés par les notes mais sont suivis par des appréciations, sans pour autant risquer d'échouer ou de redoubler.

Une recherche récente ${ }^{5}$ montre qu'un plus grand sentiment de satisfaction de leur scolarité existe chez les élèves de collège en 2010 qu'en 1992 (Øia 2010). En même temps, l'étude souligne une forte relation entre la réussite scolaire au lycée et la notation des élèves à la sortie du collège. Cet aspect nous semble fort intéressant par rapport au plaisir et à l'ennui chez les élèves, car c'est au niveau du collège que les élèves commencent à se détourner de l'école, pendant qu'ils sont encore dans le cadre de la scolarité obligatoire. Cette étude montre également qu'un plus grand nombre d'élèves de collège se sent à l'aise à l'école aujourd'hui qu'il y a dix ans. Un moins grand nombre d'élèves sèche les cours et le taux d'absentéisme est moins élevé.

Cette recherche montre aussi que les jeunes actuels se montrent plus disciplinés, plus obéissants vis-à-vis des règles, des autorités de tutelle et des enseignants qu'il y a dix et vingt ans, où ils faisaient plus de bruit et créaient davantage de désordre à l'école (Øia 2010). Dans une discussion menée à la suite de la présentation de cette étude, les élèves et les enseignants soulignent l'importance du rôle des parents pour la réussite des élèves et pour leur plaisir d'aller à l'école, également au niveau du collège.

S'il n'existe pas d'échec scolaire des élèves à la sortie du collège, et si tous les élèves ont le même droit de choisir entre les filières - générales, techniques ou professionnelles -, une étude menée par le Bureau national des statistiques (SSB) de Norvège pour l'Organisation nationale des employeurs norvégiens (NHO) montre qu'il existe de grandes différences entre les notes de base $^{6}$ obtenues par les élèves dans les différentes filières. Alors que $45 \%$ des élèves de la filière construction avaient obtenu une note de base inférieure à 3 à la fin de la scolarité obligatoire, seuls $3 \%$ des élèves des filières de l'enseignement général sont entrés au lycée avec une note de base aussi faible.

Le rapport montre ensuite que, chaque année scolaire, environ $3 \%$ des élèves sortent du collège avec $0 \%$ de points de base ${ }^{7}$. Pourtant, ces élèves «sans notes » ont le même droit à une place au lycée que les autres jeunes sortant du collège. Derrière ces faibles notes de base, il y a beaucoup d'élèves qui ne se sentent pas bien à l'école, ne s'y plaisent pas et s'y ennuient souvent. C'est sans doute l'une des premières causes d'absentéisme au collège. Mais il resterait à mener beaucoup de recherches sur ce point.

5. L'étude a été effectuée en 2010 à la demande du ministère de l’Éducation. Les résultats sont basés sur les données recueillies en 1992, 2002 et 2010.

6. L'échelle des notes de l'enseignement en Norvège va de 1 à 6, 6 étant la meilleure note. La note de base est une mesure commune permettant de comparer les notes des élèves sortant de l'école de base. La note est la somme de toutes les notes dans les matières, divisée par le nombre total de matières, calculée avec deux décimales et multipliée par 10.

7. Les élèves qui manquent de notes dans plus de la moitié des matières enseignées en sortant du collège, par exemple en raison d'un trop petit nombre de devoirs rendus pendant l'année scolaire, un taux d'absence élevé ou des dérogations fréquentes, sortent du collège avec une note de base de o \%. 
Les employeurs d'Oslo s'inquiètent de ne pouvoir recruter leurs futurs ouvriers parmi les meilleurs élèves et souhaitent une année supplémentaire en entreprise avant de commencer le lycée, ce qui est vivement contesté par les autorités ministérielles. Ces constats peuvent indiquer qu'il existe un problème sérieux, au collège comme au lycée, malgré toutes les bonnes intentions exprimées au niveau des objectifs ainsi que dans le curriculum en train d'être mis en place.

\section{Le lycée}

Dans le lycée ${ }^{8}$ norvégien, tous les élèves sortant du collège, qu'ils aient des notes passables ou pas, ont le droit légal d'avoir une place pendant trois ans pour étudier au lycée. Cet enseignement est composé de trois éléments : l'enseignement général, les spécialités de la filière et le projet d'approfondissement pour la formation dans les filières techniques et professionnelles.

Des recherches nationales montrent, par exemple, qu'en moyenne un tiers des élèves, sur l'ensemble de l'enseignement dispensé au lycée, sort du lycée sans terminer le parcours; dans les filières techniques et professionnelles, ce taux peut grimper jusqu'à $50 \%$ des élèves, dans certaines filières. Des recherches montrent aussi qu'un certain nombre d'élèves manquent de motivation pour apprendre un métier, qui ne commence pas avant la deuxième année au lycée, après une première année obligatoire avec l'introduction à toute une "famille " de métiers de la filière en question, sans autre possibilité d'accès à la formation directe de leur métier favori au départ. La situation n’aide pas à ce que les élèves se plaisent à l'école.

Les plus grands problèmes de motivation identifiés au niveau du lycée se situent dans les filières techniques et professionnelles, car il existe une nette différence entre les résultats obtenus par les élèves de ces filières et ceux des élèves qui choisissent l'enseignement général. On dirait que les élèves s'autosanctionnent, car leur accès au lycée ne se fait pas sur la base des notes obtenues, mais sur celle de leur choix personnel (Tarrou 1999).

Un exemple positif de pratiques pédagogiques dépassant la dichotomie classique entre enseignement général et enseignement technique et professionnel d'une part, et entre théorie et pratique d'autre part, peut être observé au sein de la filière construction du plus grand lycée d'Oslo. Cet exemple montre comment l'enseignement des mathématiques a été intégré au sein de la formation

8. Le lycée norvégien, qui comporte trois années de scolarité, est un droit légal pour les élèves entre 16 et 20 ans. Pour les filières générales, les études se poursuivent sur trois années scolaires en matières générales. Pour les filières techniques et professionnelles, le certificat d'études, qui qualifie également l'élève comme ouvrier qualifié, est délivré sur la base de deux années au sein de la structure scolaire et d'une année d'apprentissage en entreprise. Pour ces filières techniques et professionnelles, l'enseignement est le plus souvent organisé selon un modèle intitulé " $2+2$ », ce qui veut dire que l'ensemble de l'enseignement et la formation, qui est qualifiante, est délivré par les autorités scolaires départementales. Le plus souvent, l'organisation de ce parcours en entreprise est étalée sur deux ans, pour permettre aux entreprises de profiter de la participation des jeunes à leur activité de production. Le lieu de l'apprentissage ainsi que le formateur doivent être acceptés par les autorités scolaires et l'apprenti a droit à un accord qui décrit ses obligations et ses droits. 
pratique sur les objets, dans l'atelier scolaire de la filière construction. Beaucoup d'élèves déclarent qu'ils trouvent normal d'apprendre les mathématiques quand il s'agit des planches, des dalles, des diamètres et des angles. Plusieurs élèves qui ont obtenu une note de base de 0 choisissent justement la filière construction.

Les enseignants de ce lycée disent que, ces dernières années, ils se sont sentis obligés de penser autrement leur enseignement du norvégien, de l'anglais et des mathématiques. Au lieu de parler en termes de disciplines générales, ils intègrent les connaissances du norvégien, de l'anglais et des mathématiques à l'apprentissage des métiers dans les ateliers scolaires. Ils soulignent qu'il ne s'agit pas là d'une baisse des exigences en ce qui concerne la maîtrise attendue des élèves dans ces matières, mais d'une autre approche des connaissances générales, plus motivante pour les élèves, qui favorise davantage le sens des apprentissages et crée davantage de plaisir, moins d'ennui et moins d'angoisse. Un élève s'exclame que c'est beaucoup plus facile d'apprendre les maths quand l'enseignement est plus pratique et qu'il comprend de quoi il s'agit. Nous y reviendrons plus loin.

\section{L'APPORT DES RECHERCHES}

Ce questionnement sur le plaisir et l'ennui à l'école norvégienne montre un certain nombre de contradictions dans la société norvégienne, ce dont témoignent les débats publics et les recherches récentes.

De plus en plus d'études en Norvège ont récemment souligné le rapport entre le niveau d'études des parents d'élèves et les résultats des élèves sortant du lycée. Ces recherches montrent aussi que le manque de motivation des élèves et le "décrochage " de l'école conduisent les jeunes à connaître de sérieux problèmes plus tard dans leur vie. L'absence d'une note de base à la sortie du collège témoigne pour un élève de lacunes considérables dans ses connaissances et influe sur la poursuite de ses études ou sur le travail en entreprise. Ce signe fort d'ennui et de malaise à l'école existe même sans échec scolaire formel.

Au niveau du collège, les recherches et les débats publics dans les médias montrent qu'il existe en Norvège un problème grave en matière d'absentéisme scolaire et de sentiment de bien-être et d'épanouissement chez les élèves. En même temps, une recherche récente montre une nette amélioration du sentiment de plaisir chez les élèves de collège entre 1992 et 2010 pour ce qui concerne l'absentéisme et les conflits des élèves avec les enseignants; la même recherche met également en évidence le fait que le taux des élèves qui se sentent à l'aise à l'école a augmenté sur la même période, et qu'une bien plus faible part des élèves appréhende d'aller à l'école en 2010 qu'en 1992.

En ce qui concerne le lycée, c'est avant tout dans les filières techniques et professionnelles que le mécontentement est le plus important. Ici encore, l'insatisfaction est la plus forte dans les filières qui ont connu le plus grand changement en matière de structure et de contenu dans les deux dernières réformes. Les points le plus souvent soulevés dans les débats publics sont une 
place trop importante réservée à l'enseignement général, au cours des deux premières années de formation professionnelle au sein de la structure scolaire, et le manque de formation pratique dans les métiers spécifiques pendant la première année scolaire. Ce mécontentement se traduit par de l'absentéisme et du désintérêt dès la première année au lycée chez certains jeunes. Lorsque les élèves n'obtiennent pas les notes passables pendant ces deux premières années, le plus souvent parce qu'ils sèchent les cours, ils ont des difficultés d'obtenir des places d'apprentissage dans les entreprises, et ils ont une proposition d'une troisième année scolaire. Mais souvent ils ne la font pas. Cependant, cet échec ne se manifeste pas avant la fin de l'enseignement secondaire, lorsque l'élève n'obtient pas un certificat d'études lui permettant d'avoir un travail comme ouvrier qualifié.

Une recherche publiée en 2008 sur l'absentéisme et le manque de compétences nécessaires pour obtenir un diplôme de lycée, cinq ans après l'entrée des jeunes au lycée, a montré qu'il existe une relation nette entre le niveau d'études des parents et le niveau de compétences des élèves (Markussen, Sandberg, Lødding \& Frøseth, 2008, p. 13). Ces résultats ne sont sans doute pas étonnants dans beaucoup de pays, mais en Norvège, un pays où tant de mesures ont été mises en place pour corriger les inégalités sociales et pour favoriser l'épanouissement des élèves, en leur laissant le droit de choisir ce qu'ils souhaitent apprendre, ces résultats étonnent et dérangent la politique éducative menée en Norvège.

Dans un article basé sur une recherche en cours, Tarrou et Johannesen $(2010)^{9}$, font également référence à la recherche de Markussen et al., présentant la supposition que les variables sur l'origine sociale des élèves et le niveau d'études de leur parents sont aussi importantes pour le travail et la formation des enseignants que pour la réussite des élèves. C'est également une condition pour que les élèves se plaisent à l'école.

Elles demandent ensuite pourquoi la question de l'appartenance à une classe sociale chez les élèves et le soin du bien-être de tous les élèves sont laissés aux pratiques implicites et sous-jacentes, qui reproduisent les arbitraires culturels pendant le parcours d'études Elles soulignent que, vue sous cet angle, l'étude de Markussen et al., constitue un bon cadre de référence pour demander de nouvelles recherches et un approfondissement, basés sur des recherches empiriques.

Une autre recherche ${ }^{10}$ récente intitulée "Capital social et autres capitaux chez les enfants et les jeunes en Norvège ${ }^{11}$ ", montre le besoin d'une

9. Hedvig Skonhoft Johannesen prépare une thèse ( $\mathrm{PhD}$ ) au Centre d'études supérieures d'Akershus (AUC), au sein d'un projet de recherche intitulé " Développement des connaissances en didactique des métiers à enseigner dans les filières techniques et professionnelles et la mise en place d'un nouveau curriculum 2007-2011) » et dirigé par Anne-Lise Høstmark Tarrou. Sa thèse sur l'importance des codes culturels pour la mise en place du nouveau curriculum analyse la façon les enseignants de trois filières techniques et professionnelles mettent en place « La promotion des connaissances dans leur enseignement » (travail en cours).

10. La recherche comprend sept articles et l'article de Moshuus a étudié les enfants immigrés d'Oslo, qui se sentent marginalisés dans la société norvégienne.

11. Le rapport « Capital social et autres capitaux chez les enfants et les jeunes en Norvège » est publié par l'Institut norvégien pour la recherche sur la croissance, l'épanouissement et le vieillissement (NOVA). 
meilleure compréhension à l'école de la «culture juvénile » et d'une ouverture aux apprentissages informels extérieurs à l'école. Cette recherche met l'accent sur le fait que " l'État providence ", en Norvège, ouvre des possibilités aux jeunes à capital social faible en termes de confiance, de normes sociales et de réseaux sociaux caractérisés par la réciprocité et l'engagement. L'étude sur les enfants immigrés d'Oslo qui se sentent marginalisés montre que ces jeunes jouent souvent sur leur différence par rapport à la majorité des autres jeunes en se créant une "identité des rues" (Moshuus 2011). Selon Moshuus, il est fréquent de penser que les institutions publiques ne jouent pas un rôle important pour les jeunes marginalisés. Mais il souligne au contraire que le manque de capital culturel chez les jeunes marginalisés à Oslo conduit à ce qu'ils obtiennent un contact plus étroit avec les institutions de l'État providence autres que l'École, et que ce contact peut faciliter pour eux la possibilité de devenir membres de groupes marginaux dans la société norvégienne (Moshuus 2011).

\section{RETOUR SUR LES QUESTIONS SOULEVÉES}

Le poids très lourd de l'enseignement général pendant les deux premières années de l'enseignement au lycée est considéré par les hommes politiques et par les partenaires sociaux comme le facteur qui empêche le plus les élèves de commencer l'apprentissage d'un métier dès la première année de lycée. L'élève qui sort du collège avec l'envie de devenir coiffeur, ne peut par exemple, dans la plupart des lycées, pas commencer l'apprentissage de son métier tout de suite, mais doit d'abord faire une première année d'introduction à toute une famille de métiers et continuer en même temps à suivre une formation générale, avant de commencer à coiffer. Mais il faudrait se demander si l'apprentissage de la théorie, tel qu'il se pratique dans les lycées aujourd'hui, est bien mené. L'internationalisation du marché, la mobilité et les nouvelles technologies exigent aujourd'hui des jeunes et de leurs enseignants un niveau d'abstraction plus élevé qu'il y a quelques années. C'est pourquoi il ne me semble pas opportun de réduire l'apprentissage des matières générales (Tarrou 2009, Tarrou et Johannesen 2010) mais il y aurait lieu de les mettre en perspective autrement, pour permettre aux élèves de comprendre de quoi il s'agit comme le disait justement l'un d'eux, dans l'exemple cité plus haut.

Ce que montrent le débat public et les quelques recherches présentés ici, c'est pourtant la réalité de problèmes complexes pour les élèves comme pour les enseignants, qui persistent malgré toutes les bonnes intentions exprimées et toutes les mesures prises pour les résoudre.

L'une des questions les plus importantes me parait être celle de l'inclusion des élèves issus de milieux socioculturels autres que le milieu dominant à l'école, celui qui impose l'ordre du jour de ce que les élèves doivent apprendre au cours de leur scolarité. Comment réintroduire la joie dans la vie scolaire de ces élèves? 
Comment se fait-il que, dans un système scolaire aussi ouvert que le système norvégien, avec une si grande liberté laissée aux élèves pour choisir leur voie d'études et leur mode d'apprentissage, ceux qui font les meilleurs choix pour leur carrière future soient toujours les élèves qui ont les meilleurs conseillers à la maison?

Malgré toutes les mesures mises en place en Norvège pour que les élèves se sentent à l'aise à l'école, il existe toujours des problèmes sérieux et non résolus. Même si l'échec scolaire n'existe pas sur toute la scolarité des jeunes jusqu'à vingt ans, qu'il n'existe pas de redoublement, qu'il n'y a pas de notation avant la dernière année du collège, que l'enseignement est un droit pour tous les jeunes jusqu'au lycée inclus, les problèmes existent toujours pour une partie des jeunes qui n'éprouvent pas de plaisir à aller à l'école, s'y ennuient ou s'y sentent angoissés.

\section{BIBLIOGRAPHIE}

Kunnskapsdepartementet (2008) : Fagopplaring for framtida. Norges offentlige utredninger NOU 2008:18.

MARKUSSEN E., SANDBERG N., LØDDING B., \& FRØSETH M. W. (2008) : Bortvalg og kompetanse: gjennomføring, bortvalg og kompetanseoppnåelse $i$ videregående opplaering blant 9749 ungdommer som gikk ut av grunnskolenpå Østlandet våren 2002: hovedfunn, konklusjoner og implikasjoner fem år etter (Vol. 13/2008). (Refus de cours et compétences : parcours, refus, acquisition de compétences au lycée parmi 9749 jeunes sortant de l'enseignement de base en 2002.) Oslo: NIFU STEP.

MOSHUUS, GEIR (2011) : Sosial kapital og andre kapitaler hos barn og unge i Norge (Capital social et autres capitaux chez les enfants et les jeunes en Norvège). L'Institut norvégien pour la recherche sur la croissance, la providence et le vieillissement (NOVA). Oslo. 2010.

TARROU A-L. H. \& JOHANNESEN H.S. (2010) : Perspektiver på kunnskapssyn ved læring i yrkesfagopplæringen. (Perspectives sur savoirs et apprentissages dans l'apprentissage aux métiers). I. Aamotsbakken, B. Laring og medvirkning (Apprentissage et participation). (Red.), p. 93-107. Oslo. Universitetsforlaget.

TARROU A-L. H. (2009) : Hur anställningsbar är man efter genomgången norsk yrkesutbildning? I. Berglund, G. \& Fejes, A. Anställningsbarhet. Perspektiv från utbildning och arbetsliv, p. 103-120. Lund. Studentlitteratur.

TARROU A-L. H. (2003) : "La formation professionnelle initiale en Norvège. Entre valeurs démocratiques et partenariats économiques ». Revue internationale d'éducation de Sèvres, $\mathrm{n}^{\circ} 34$, décembre 2003, p. 127-136.

TARROU Anne-Lise Høstmark (2001) : «Inégalités dans une société égalitaire », in Politique d'éducation et de formation. Analyses et comparaisons internationales, vol. 1, $\mathrm{n}^{\circ} 2$, 2001, p. 105-122.

TARROU Anne-Lise Høstmark (1999): Inégalités des cultures professionnelles techniques et humanistes des enseignants. Presse universitaire de France. (Aspects internationaux. Pédagogie comparée). Paris 1999. 296 p.

Utdanningsdepartementet (2004) : Kultur for loering St.meld. nr. 30 (2003-2004).

ØIA, Tormod (2010) : Motivasjon, mestring og resultater (Motivation, maîtrise et résultats). Rapport de recherche. NOVA. 